\title{
Seismic vulnerability assessment of RC curved bridges piers subjected to bidirectional earthquake
}

\author{
Rui $\mathrm{Li}^{1, *}$ \\ ${ }^{1}$ CHANG'AN UNIVERSITY, highway school, 710064 Middle-section of Nan'er Huan Road Xi'an, China
}

\begin{abstract}
Curved beam bridge is a kind of irregular structure, which has the advantage of adapting to complex terrain, but it is more vulnerable to damage than regular bridge under earthquake. This paper investigates the vulnerability of curved continuous girder bridges under the action of bidirectional earthquake horizontal earthquake, and the difference of vulnerability between side pier and intermediate pier is analyzed. Fragility assessment is performed using an incremental dynamic analysis method subjected to a wide range of as-recorded sequences. A proper engineering demand parameter (EDP) which can result in the most probability of failure at bridges employed in this study is determined. Result indicates that only considering the unidirectional seismic input will underestimate the seismic response and potential damage of the structure, which is not accurate for the seismic performance evaluation of the bridge. Result also shows that the damage probability of the intermediate pier of the curved bridge is higher than that of the side pier, and the more serious the failure, the smaller the difference between the two piers.
\end{abstract}

\section{Introduction}

In the Wenchuan earthquake, the large relative displacement at the expansion joint of Baihua bridge caused the upper structure of the fifth continuous beam (with a radius of curvature of $66 \mathrm{~m}$ ) to fall and break, resulting in the overall collapse of the structure[1]. For the four circular ramp bridges of Huilan interchange, the piers close to the abutment are rigidly connected with the upper structure. Under various actions, bending shear failure occurs. The damaged piers present $\mathrm{X}$-shaped fracture section, the longitudinal bars at the bottom of the piers buckle, and the confined concrete is crushed[2]. The seismic research of curved bridges began in 1971 when the San Fernando earthquake in Los Angeles caused the fall of two curved bridges on the interstate highway interchange. [3] and [4] respectively carried out detailed modeling analysis and shaking table test for the damaged double curve girder bridge, and put forward some suggestions for seismic design and calculation of curved bridge. The influence of curve radius and other factors on the seismic performance of curved bridges is studied by means of spectrum analysis or time history analysis[5] [6]. [7] studied the influence of curve radius on the damping effect of viscous damper of curved bridge.

In the seismic research of curved beam bridge, the actual input direction of seismic wave will have different angles with different Bearings, so there is no clear main direction to guide the seismic wave input to obtain the most unfavorable response of curved beam bridge. In the past, many researchers have discussed the problem of the most unfavorable direction. [8] used the rotation method to input one-way time history of a curved continuous beam bridge with $0-180$ degrees every 10 degrees as an excitation direction, and obtained the maximum response angle of the middle pier in radial and tangential directions respectively. [9] gives the formula for calculating the most unfavorable input angle of curved girder bridge, and the difference between the calculation results and the rotation method is small. However, no matter what method is used, most of the conclusions are focused on the most unfavorable input angle of a certain bridge pier in a certain direction. Different focuses on the bridge often lead to different worst input angles. It is difficult to evaluate how to input seismic wave to make the bridge structure suffer the most adverse overall stress. With the development of performance-based seismic design method, the seismic performance of structural components in a specific site can be evaluated from the perspective of probability, and the seismic performance of the whole bridge structure can also be calculated. Therefore, taking the whole bridge vulnerability characteristics of curved girder bridge as the evaluation standard, a seismic input direction which makes the curved beam bridge most vulnerable to damage can be determined.

Curved beam bridge is a kind of irregular structure, which has the advantage of adapting to complex terrain, but it is more vulnerable to damage than regular bridge under earthquake. For the straight-line bridge, the most unfavorable response value of the straight-line bridge can be obtained when the seismic wave direction and the bridge axis direction are 0 degree and 90 degree respectively. However, for curved bridge, the direction of seismic wave is not the same as the angle of main beam in different positions. Therefore, the curved bridge does

\footnotetext{
* Corresponding author: 13572500825@163.com
} 
not have a certain longitudinal (transverse) direction, and it is necessary to consider bidirectional seismic input. This paper aims to evaluate the fragility curves of piers of curved bridges subjected to bidirectional earthquake.

\section{Model}

A continuous curved beam bridge has been considered in this study. With a total length of approximately $100 \mathrm{~m}$, this four-span RC bridge comprises a continuous reinforced concrete multi-cell box girder deck supported on two abutments at both ends and three single column bents (piers) at Mid-spans. The axis of the bridge is a circular curve with a radius of curvature of $80 \mathrm{~m}$. The height of columns is approximately $10 \mathrm{~m}$ with a circular crosssectional geometry which is constant along the length of column with $1.6 \mathrm{~m}$-diameter. This pier is consisted of 30 longitudinal bars, $28 \mathrm{~mm}$-diameter and $12 \mathrm{~mm}$-diameter transverse reinforcement in the form of spiral. The cross section the bridge has been shown in Fig. 1.

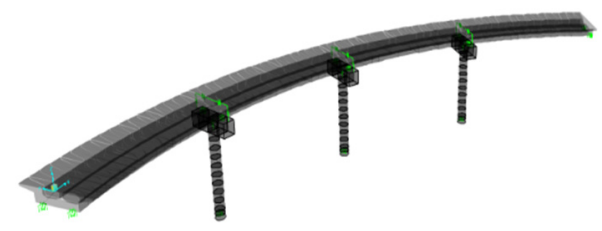

Fig.1 Three dimensional model of curved bridge

Three-dimensional non-linear finite element models (FEM) are established in SAP2000. The RC deck has been modeled as an elastic straight beam column element; it is assumed that superstructure behaves elastically during the earthquake according to the assumption presented by[10]. The plate rubber bearing is simulated by plastic (Wen) connection unit. The bilinear model with yield point as the only turning point is chosen to simulate the plastic hinge in bridge piers, and the simplified skeleton curve is as shown in Fig. 2. Multiline plastic connection unit can be selected to simulate it. For curved beam bridge, the pier will be damaged in both transverse and longitudinal directions, so two bending degrees of freedom are set as non-linear, three translation and torsion are set as fixed.

\section{Ground motion}

The acknowledgements should be typed in 9-point Times, without title. Assuming that the soil type in this paper is a class II type, 10 seismic records are randomly selected after the near-field seismic waves are excluded from the Pacific earthquake engineering research center (PEER) database according to the shear wave velocity. Based on the statistics of the peak acceleration of the bidirectional component of a large number of seismic waves, [11] suggests that a seismic wave can be input into a bidirectional seismic wave at a peak acceleration ratio of 1:0.85. However, the as-record data show that the spectral characteristics of two horizontal components of the same seismic wave sometimes differ greatly. If the difference is ignored in the calculation, the calculation results are often quite different from the actual ones. Therefore, in this paper, when inputting bidirectional seismic waves, two actual seismic wave components are selected without adjustment, which is consistent with the as-record earthquake. The acceleration of the selected seismic wave is scaled in $0.1 \mathrm{~g}$ increments to make its peak value from $0.1 \mathrm{~g}$ to $1.0 \mathrm{~g}$, which is divided into 10 levels, with 100 seismic waves in total. Fig. 3 shows the initial response spectrum curves, respectively.

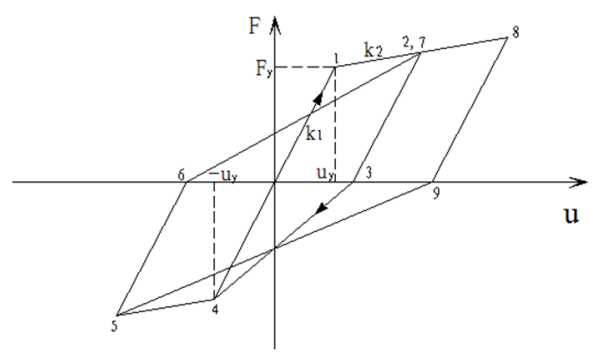

Fig.2 bilinear model curve

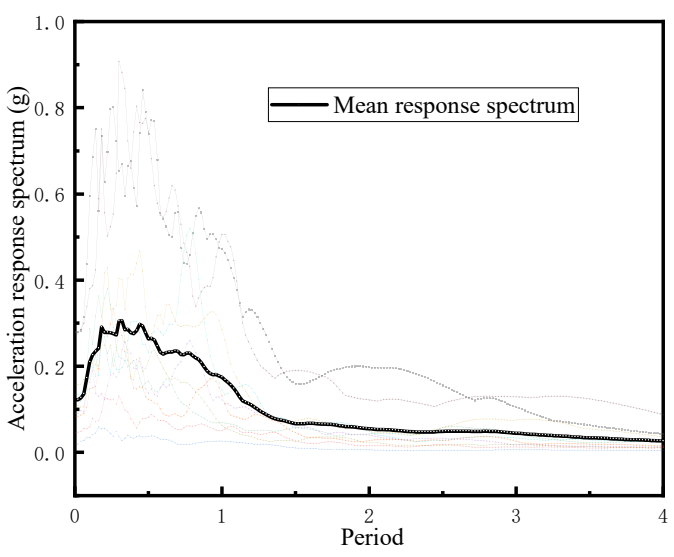

Fig.3 Initial response spectrum

\section{Method}

Fragilities can be calculated from the median and logstandard deviation values, with the assumption of lognormal distribution. The equation for this function is given by:

$$
P_{f}=\phi\left[\frac{\ln \left(\mathrm{a}(\mathrm{IM})^{b}\right)-\ln (L S)}{\sqrt{\beta_{E D P}^{2}+\beta_{L S}^{2}}}\right]
$$

where $\mathrm{P}$ is the probability of exceedance of the limit state based on ground motion intensity (IM), $\Phi$ is the probability density function of a normal distribution, IM is the ground motion intensity indexes, such as peak ground acceleration (PGA) or spectral acceleration, LS is limit value of damage index for each failure state of structure, EDP is engineering demand parameter, $\beta_{E D P}$ and $\beta_{L S}$ are the log-normal standard deviation of structural seismic response and limit state of components, respectively; $a$ and $b$ are fitted parameter values. When SA 
is used as vulnerability analysis index, a is 0.4 , and when PGA is used as index, a is 0.5[12].

Curved beam bridge is also an irregular bridge, the influence of high-order vibration mode on the structural response cannot be ignored, so EDP defined by curvature ductility ratio for pier can more accurately reflect the deformation and failure of materials in the plastic hinge area of pier.

Considering the complexity of the structure and the small basic period, PGA, which is simple in concept, can be selected as the seismic intensity index to meet the calculation requirements.

\section{Fragility curves of piers}

This section compares the response difference of piers under unidirectional seismic excitation. Seismic excitation direction and bridge arrangement are shown in Fig. 4. According to the difference of seismic input direction, three working conditions are established.

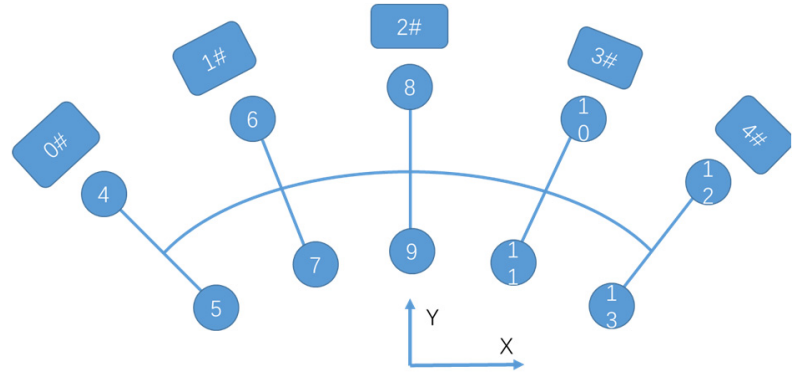

Fig.4 Schematic diagram of seismic wave input direction

Case 1 (longitudinal direction): the larger component of seismic wave will be input along $\mathrm{X}$ direction.

Case 2 (transverse direction): the smaller component of the seismic wave will be input along the $\mathrm{Y}$ direction.

Case 3 (bidirectional): the larger component of seismic wave along $X$ direction and smaller component of seismic wave along $\mathrm{Y}$ direction, bidirectional input.

The comparison of the maximum curvature of $1 \#$ pier bottom and the fragility curves of $1 \#$ and $2 \#$ pier subjected to bidirectional earthquake are shown in Fig. 5 and Fig. 6 respectively.

It can be seen from Fig. 5 that with the increase of PGA, the comprehensive maximum curvature of pier bottom increases continuously under three cases. When the comprehensive maximum curvature reaches the limit value of slight damage, the increase speed of the comprehensive maximum curvature value increases significantly, because the plastic hinge area of the pier has been yielding and entered the plastic deformation stage. Before PGA reaches $0.7 \mathrm{~g}$, the maximum curvature of the pier under the condition of transverse input and longitudinal input is basically same. When PGA is greater than $0.7 \mathrm{~g}$, the maximum curvature under the condition of longitudinal input is gradually greater than that of the pier under the condition of transverse input. And with the increase of PGA, the curvature values of the three cases are more and more different. It can be seen that bidirectional seismic input will increase the maximum curvature of the pier bottom and increase the bending deformation of the pier.

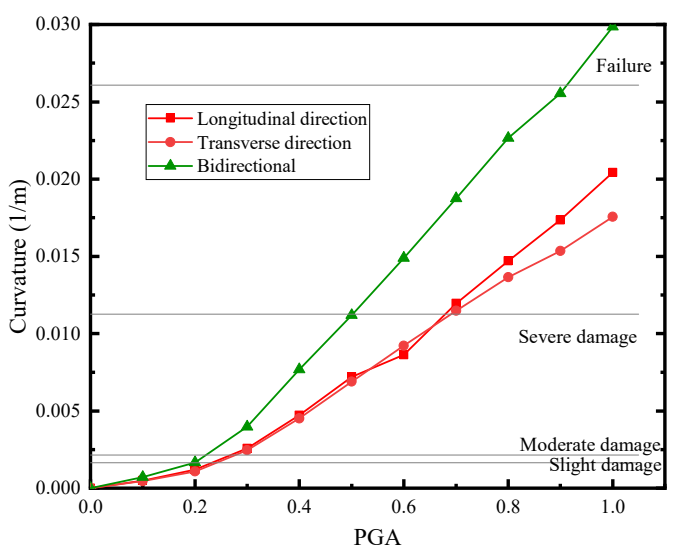

Fig.5 Comprehensive maximum curvature comparison of $1 \#$ piers

According to Fig. 6(a), the probability of bending failure of $1 \#$ pier increases with the increase of ground motion intensity. When PGA is less than $0.1 \mathrm{~g}$, the probability of failure is approximately 0 , which indicates that 1 \# pier will not be destroyed under this condition. When PGA gradually increases from $0.1 \mathrm{~g}$ to $0.4 \mathrm{~g}$, the probability of slight and moderate damage increases rapidly, and the two damages probability are very close, because the boundary value of dividing moderate failure and slight failure is very close, and the difference of curvature ductility coefficient is only 0.24 . When PGA reaches $0.4 \mathrm{~g}$, the probability value of slight and moderate damage is close to 1 , indicating that slight and moderate damage are inevitable, while the probability of severe damage and failure is still low, the probability of severe damage is 0.118 and failure is 0.002 . With the further increase of PGA, the probability of slight and moderate damage tends to be stable to more than 0.99 , and the probability of severe damage continues to increase, but the growth rate is significantly lower than that of slight and moderate damage. The growth rate of probability of failure is slower, indicating that the sensitivity of severe damage and failure to PGA growth is far lower than that of slight damage and moderate damage. The fragility curve of bending failure of 2\#pier is similar to that of $1 \#$ pier. It is worth noting that the axial pressure at the bottom of the $2 \#$ pier is smaller than that of the $1 \#$ pier, so the curvature ductility coefficient of the $2 \#$ pier will be relatively large, resulting in the failure probability of the $2 \#$ pier is higher than that of the $1 \#$ pier, which shows that the failure of the $2 \#$ pier will be more serious under the action of bidirectional seismic wave.

The probability difference of $1 \#$ pier and $2 \#$ pier under each damage state is shown in the Fig. 7. The difference of four kinds of damage from slight damage to failure first reaches a peak state with the increase of PGA, and then decreases. The probability difference of four kinds of damage states reaches the maximum with the increasing of corresponding PGA in turn. This is because with the increase of PGA, the growth rate of failure probability of each level gradually increases to a peak value, then decreases and finally approaches to zero infinitely, and 
The difference between the responses of $1 \#$ pier and $2 \#$ pier leads to the non-synchronization of the probability growth of the same damage state.

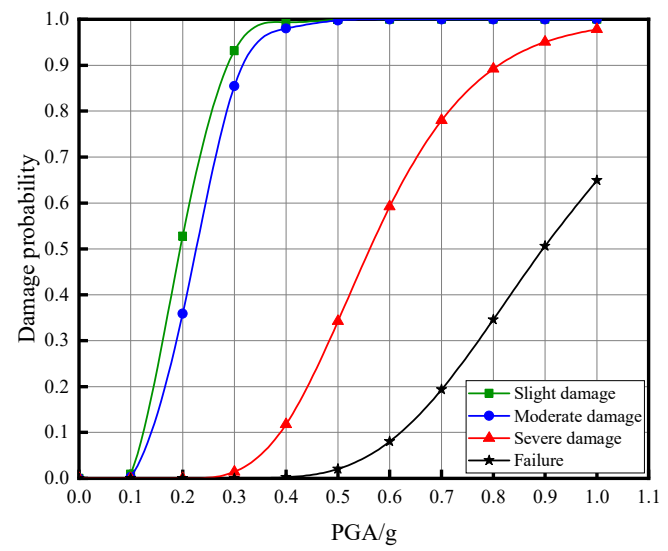

Fig. 6(a) Bending failure fragility curve of 1\# pier

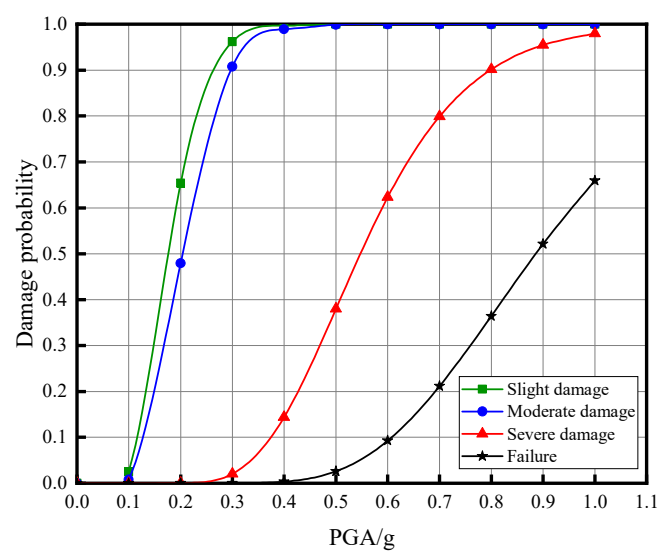

Fig. 6(b) Bending failure fragility curve of 2\# pier

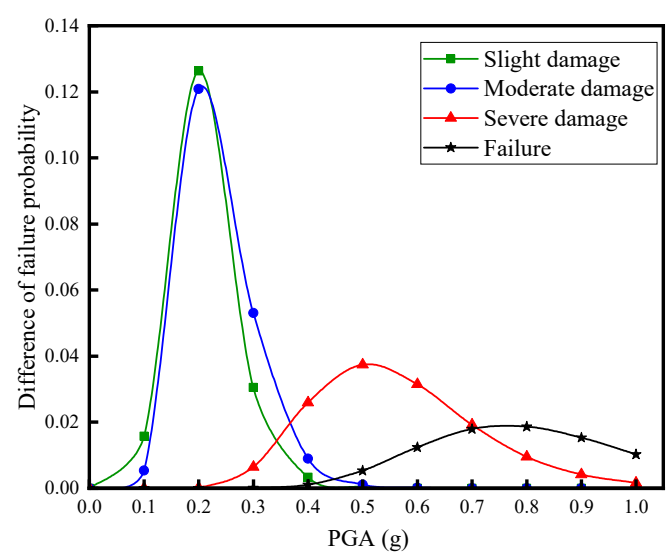

Fig.7 Difference of bending failure probability between $1 \#$ pier and $2 \#$ pier

According to Fig. 8, the probability of each damage state of $2 \#$ pier in bidirectional seismic input is significantly higher than that in unidirectional input, in which the probability values of longitudinal input and transverse input are very close, but the longitudinal input is slightly higher than that of transverse input. For the two cases of slight damage and moderate damage, the damage probability value of the bridge pier increases rapidly to 1 under unidirectional input and bidirectional input. It is worth noting that with the increase of ground motion intensity, the increase degree of bidirectional input to the probability of slight, moderate and severe damage increases first and then decreases compared with unidirectional input, while for failure, the greater the ground motion intensity is, the greater the aggravating effect of bidirectional earthquake is. For the VIII seismic fortification area, the probability of bridge pier at all damage states is less than $1 \%$ under the condition of E1 earthquake intensity. The probability of severe damage and failure of bridge pier under the condition of E2 earthquake intensity is less than $1 \%$, and compared with the horizontal input, the probability of slight and moderate damage increases by $28 \%$ and $35 \%$ respectively. For the IX seismic fortification area, the possibility of severe damage and failure of piers is ignored under the condition of E1 earthquake intensity. Compared with the transverse input, the bidirectional input increases the probability of slight and moderate damage by $31 \%$ and $22 \%$ respectively. Under the condition of E2 earthquake intensity, the probability of slight, moderate, severe damage and failure of pier is increased by $0.3 \%, 1.3 \%, 31 \%$ and $3.3 \%$ respectively by bidirectional input compared with transverse input.

\section{Conclusion}

In this paper, a four-span curved continuous beam bridge is taken as an example to compare and analyze the structural response difference under different cases of unidirectional and bidirectional seismic input. The fragility curve of bridge pier under bidirectional seismic input is calculated by the method of probability seismic demand analysis based on IDA, and the development rule and difference of damage probability under bidirectional and unidirectional seismic input are compared and studied.

In the case of bidirectional seismic input, the damage probability of bridge pier is $0-40 \%$ higher than that of unidirectional input. With the increase of ground motion intensity, for the damage state other than failure, the aggravating effect increases first and then decreases. For failure state, the effect will become more and more severe with the increase of ground motion intensity. In high intensity area, the increasing effect of bidirectional seismic input on the probability of severe damage and failure state is more obvious. Therefore, only considering the unidirectional seismic input will underestimate the seismic response and potential damage

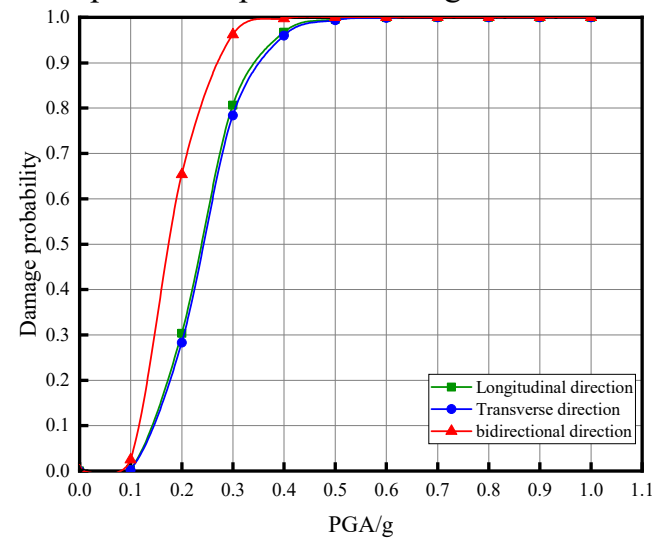

(a) Slight damage 


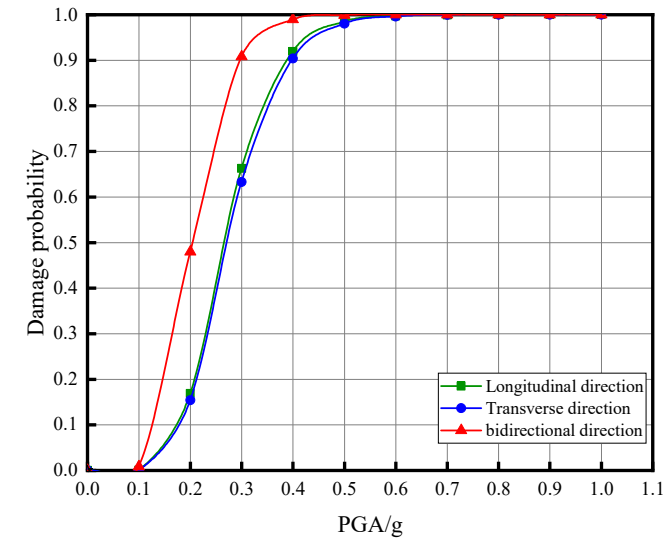

(b) Moderate damage

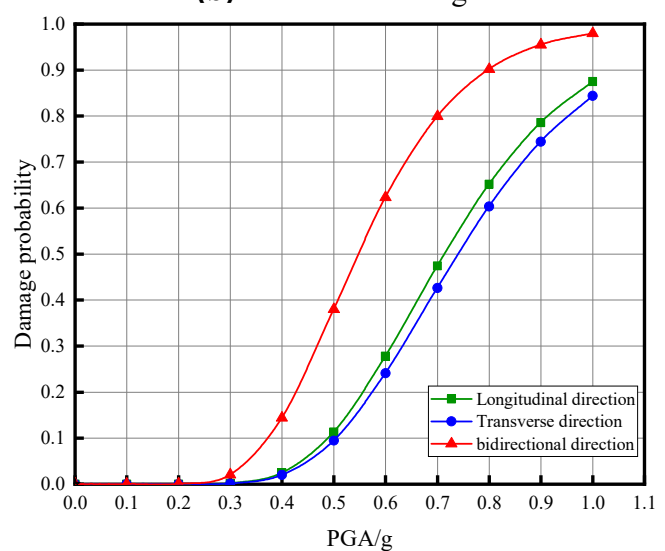

(c) Severe damage

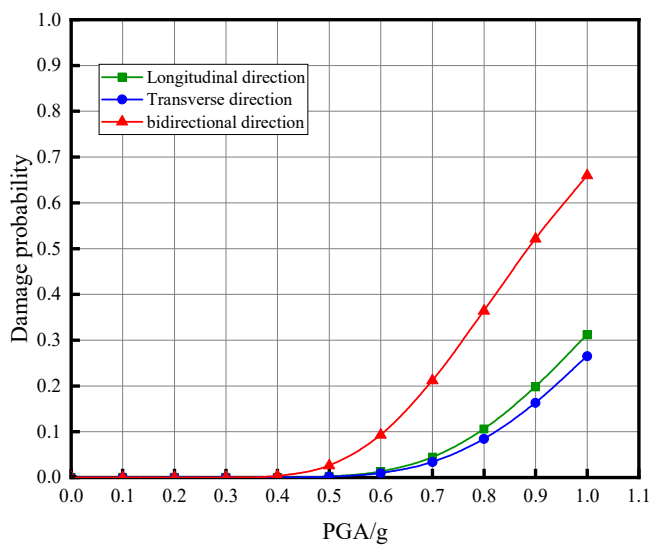

(d) Failure

Fig.8 Vulnerability comparison of unidirectional and bidirectional seismic input of $2 \#$ pier

of the structure, which is not accurate for the seismic performance evaluation of the bridge.

The damage probability of the intermediate pier of the curved bridge is higher than that of the side pier, and the more serious the failure, the smaller the difference between the two piers. When the ground motion intensity is less than $0.4 \mathrm{~g}$, the probability of slight and moderate damage of the intermediate pier is $13 \%$ higher than that of the side pier; when the seismic intensity is more than $0.4 \mathrm{~g}$, the probability of slight and moderate damage of the intermediate pier and the side pier is basically the same, but the probability of severe damage of the intermediate pier is less than $4 \%$, and the probability of failure of the intermediate pier is less than $2 \%$.

\section{References}

1. Ren, L., Characteristics of bridge damages in Wenchuan earthquake. Journal of Nanjing University of Technology, 2009.

2. Li, J., T. Peng, and Y. Xu, Damage investigation of girder bridges under the Wenchuan earthquake and corresponding seismic design recommendations. Earthquake Engineering and Engineering Vibration, 2008. 7(4): p. 337-344.

3. Tseng, W.S. and J. Penzien, Seismic analysis of long multiple-span highway bridges. Earthquake Engineering \& Structural Dynamics, 1975. 4(1): p. 124.

4. Williams, D. and W. Godden, Seismic response of long curved bridge structures: Experimental model studies. Earthquake Engineering \& Structural Dynamics, 1979. 7(2): p. 107-128.

5. Zhu, D.S., S. Liu, and L.S. Yu, Research on Seismic Response of Curved Girder Bridges. China Journal of Highway and Transport, 2002. 15(3): p. 42.

6. Yongliang, Z., Influence of curvature radius change on seismic response of urban curved bridge. World Earthquake Engineering, 2012.

7. Xingjun, Q.I., Influence of Variation of Radius of Curvature on Seismic Mitigation Effectiveness of Viscous Dampers for Curved Girder Bridge. Journal of Highway and Transportation Research and Development, 2012.

8. Junyan, W., Nonlinear Seismic Responses Research of Curved Girder Bridge. Dalian University of Technology, 2016.

9. Quan, W., Studies on Seismic Analysis of Large-span Bridges Subjected to Multi-component and Multiplesupport Earthquake Excitations. Dalian University of Technology, 2008.

10. TG/TB02-01, Guidelines for seismic design of highway bridges, Beijing, China. 2008.

11. GB, "Code for seismic design of buildings." GB50011-2010, Beijing, China. 2010.

12. HAZUS99, F.E.M.A., Earthquake Loss Estimation Methodology: User's Manual. Federal Emergency Management Agency, Washington DC. 1999. 